\title{
On Efficient Bandwidth Allocation and Call Admission Control for VBR Service Using UPC Parameters*
}

\author{
Dapeng Wu and H. Jonathan Chao
}

Department of Electrical Engineering, Polytechnic University, New York, USA

\begin{abstract}
Provision of Quality-of-Service (QoS) guarantees is an important and challenging issue in the design of Asynchrous Transfer Mode (ATM) networks. Call Admission Control (CAC) is an integral part of the challenge and is closely related to other aspects of network design such as traffic characterization and QoS specification. Since the Usage Parameter Control (UPC) parameters are the only standardized traffic characterizations, developing efficient CAC schemes based on UPC parameters is significant for the implementation of CAC on ATM switches. In this paper, we develop a CAC algorithm called TAP (TAgged Probability) as well as two other CAC algorithms using the UPC parameters. These CAC algorithms are based on our observation that the loss-probability-to-overflow-probability ratio tends to decrease as the number of sources increases. The loss-probability-to-overflow-probability ratio $K$ sheds light on increasing resource utilization while still guaranteeing QoS. Analysis, simulation, and numerical results have shown that the proposed TAP algorithm is simple and efficient. Therefore, we recommend the TAP algorithm for CAC on high-speed ATM switches.
\end{abstract}

Key Words: CAC, VBR, UPC, Loss-probability-to-overflow-probability Ratio, Effective Bandwidth

${ }^{*}$ The work described in this paper is supported by Sumitomo Electric Industries, NYNEX, and NY State Science and Technology Foundation. 


\section{Introduction}

The Asynchronous Transfer Mode (ATM) network is believed to be capable of supporting various services with drastically different traffic characteristics and quality-of-service (QoS) requirements. The deployment of integrated services with a broad range of burstiness characteristics and their integration through statistical multiplexing have focused a great deal of attention on Call Admission Control (CAC) as one of the prime instruments of rate-based congestion control for the last decade.

To facilitate CAC and policing, the ATM Forum standardized Usage Parameter Control (UPC) parameters, through which the users must declare their traffic characteristics [1]. The source traffic can be characterized by the UPC descriptors $\left(\lambda_{p}, \lambda_{s}, B_{s}\right)$, i.e., Peak Cell Rate (PCR) $\lambda_{p}$, Sustainable Cell Rate (SCR) $\lambda_{s}$, and the Maximum Burst Size (MBS) $B_{s}$.

Many theoretical CAC schemes are not implementable because the parameters they need cannot be provided by the current ITU-T (International Telecommunication Union - Technical Committee) or ATM Forum standards. Different from these theoretical efforts, our CAC schemes are based on UPC parameters to be consistent with ATM Forum standards and they are implementable. In this paper, we develop a simple and efficient CAC algorithm called TAP (TAgged Probability) as well as two other algorithms. These CAC algorithms are used for Variable Bit Rate (VBR) service only. The QoS metric considered here is cell loss ratio.

The paper is organized as follows. Section 2 describes the existing CAC schemes that use UPC parameters. Section 3 introduces our CAC schemes. Through simulations and numerical evaluation, we compare the performance of different CAC schemes of interest in Section 4. We draw our conclusions in Section 5.

\section{Existing CAC Schemes Based on UPC Parameters}

A number of CAC schemes have been proposed [2-11]. Since our CAC schemes are based on effective bandwidth, Lucent's CAC, and NEC's CAC, we summarize the key points of these schemes in this section. We first describe a worst-case traffic model these CAC schemes will use.

\subsection{Worst-Case Traffic Model}

If only the UPC parameters $\left(\lambda_{p}, \lambda_{s}, B_{s}\right)$ are known, a way to ensure a CAC algorithm works accurately is to assume the actual traffic is as bad for performance as it could possibly be, given the parameter values. Therefore, worst-case traffic modeling becomes a hot topic for researchers [12-16].

The departure process from dual leaky buckets with the UPC parameters $\left(\lambda_{p}, \lambda_{s}, B_{s}\right)$ can be modeled as an extremal, periodic on-off process with "on" and "off" periods given by

$$
T_{\text {on }}=\frac{B_{s}}{\lambda_{p}} \quad \text { and } \quad T_{\text {off }}=\frac{B_{s}}{\lambda_{p}} \cdot \frac{\left(\lambda_{p}-\lambda_{s}\right)}{\lambda_{s}} .
$$




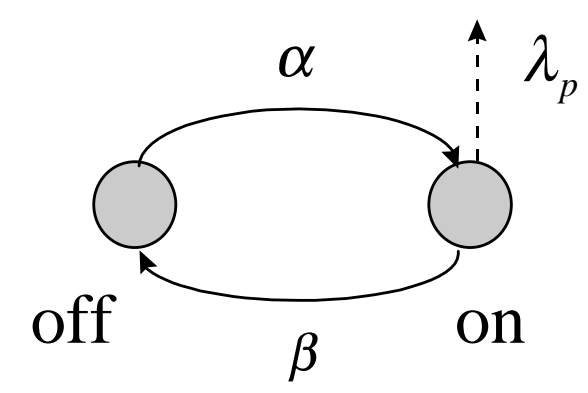

Figure 1: On-off source model.

Doshi proved this process to be the worst-case source model in the bufferless case [12]. Although Doshi gave a counter example (i.e., a three-state process) to demostrate it is not the worst-case in the buffered case, in many cases this model can be considered as the worst-case source model in the sense of maximizing steady-state cell loss probability in the ATM node [3] [12-15]. The probability such a source is in the "on" state is given by

$$
P_{o n}=\frac{T_{o n}}{T_{o n}+T_{o f f}}=\frac{\lambda_{s}}{\lambda_{p}}
$$

\subsection{Effective Bandwidth}

Hui first introduced the concept of effective bandwidth [2], which reflects the source characteristics and the service requirements. One method to calculate the effective bandwidth was given by Elwalid and Mitra [3].

The source is assumed to be either on for an exponentially distributed length of time with a mean length $1 / \beta$, when the rate is the peak rate $\lambda_{p}$, or off for an exponentially distributed interval of length $1 / \alpha$, when the rate is 0 (see Figure 1 ). If we know the UPC parameters $\left(\lambda_{p}, \lambda_{s}, B_{s}\right)$, we can obtain $\alpha$ and $\beta$ using the worst-case traffic model described in Section 2.1.

$$
\begin{aligned}
& \alpha=\frac{1}{T_{o f f}}=\frac{\lambda_{p} \cdot \lambda_{s}}{B_{s} \cdot\left(\lambda_{p}-\lambda_{s}\right)} \\
& \beta=\frac{1}{T_{o n}}=\frac{\lambda_{p}}{B_{s}} .
\end{aligned}
$$

Let sources thus characterized share a single buffer of size $B$ and require an acceptable cell loss ratio of $p$. Then we define $\eta=\log p / B$, and the effective bandwidth of the source, $e$, is given by:

$$
e=\operatorname{MRE}\left(\Lambda-\frac{1}{\eta} M\right)
$$

where 
MRE is Maximum Real Eigenvalue of the matrix;

$$
\begin{aligned}
& \Lambda=\left[\begin{array}{cc}
0 & 0 \\
0 & \lambda_{p}
\end{array}\right] ; \\
& M=\left[\begin{array}{cc}
-\alpha & \beta \\
\alpha & -\beta
\end{array}\right] .
\end{aligned}
$$

Based on the effective bandwidth, the admission criterion is satisfied if $e<c$ and violated if $e$ $>c$ ( $c$ is the available bandwidth). The effective bandwidth $e$ only depends on $(\mathrm{M}, \Lambda)$, and on the buffer size and QoS (i.e., overflow probability) through $\eta$.

\subsection{Lucent's CAC}

Based on an extremal, periodic, on-off regulated traffic model, Elwalid, Mitra, and Wentworth developed a two-phase CAC [7], which has been implemented on Lucent's GlobeView-2000 ATM switches [8]. In the first phase, they consider lossless multiplexing only. In the second phase, they analyze the effect of statistical multiplexing by using the Chernoff large derivation approximation.

The system model used in their algorithm is a network node with a buffer of size B, a link of capacity $\mathrm{C}$, and dual leaky-bucket regulated input traffic sources, each specified by parameters $\left(\lambda_{p}\right.$, $\left.\lambda_{s}, B_{T}\right)$, i.e., Peak Cell Rate (PCR) $\lambda_{p}$, Sustainable Cell Rate (SCR) $\lambda_{s}$, which is the token rate, and the token buffer size $B_{T}$. The relation between the token buffer size $B_{T}$ and the Maximum Burst Size (MBS) $B_{s}$ is given by:

$$
B_{T}=B_{s}\left(1-\frac{\lambda_{s}}{\lambda_{p}}\right)
$$

\subsubsection{Lossless Multiplexing}

They first consider the base case where the QoS requirement is zero loss. They derived the lossless effective bandwidth $e_{0}$ for each homogeneous source as follows:

$$
e_{0}=\left\{\begin{array}{cl}
\frac{\lambda_{p}}{1+\frac{B / C}{B_{T}}\left(\lambda_{p}-\lambda_{s}\right)} & \text { if } \lambda_{s} \leq \frac{B_{T}}{B / C} \\
\lambda_{s} & \text { if } \frac{B_{T}}{B / C} \leq \lambda_{s}<\lambda_{p}
\end{array} .\right.
$$

\subsubsection{Statistical Multiplexing}

In the second phase of the algorithm, a very small loss probability is allowed to extract statistical multiplexing gain from the time-varying unused portions of the resources allocated to the connections. The constraints are

$$
\operatorname{Pr}\left[\sum_{i} r_{i}(t) \geq C\right] \leq C L R \quad \text { and } \quad \operatorname{Pr}\left[\sum_{i} b_{i}(t) \geq B\right] \leq C L R
$$


where $r_{i}(t)$ is the instantaneous rate of a connection and $b_{i}(t)$ is the instantaneous buffer occupancy of a connection. Using the Chernoff bound, one can find that

$$
\operatorname{Pr}\left[\sum_{i} r_{i}(t) \geq C\right] \leq \exp \left(-F_{K}\left(s^{*}\right)\right)
$$

where

$$
\begin{aligned}
& F_{K}\left(s^{*}\right)=\sup _{s \geq 0} F_{K}(s), \\
& F_{K}(s)=s C-\sum_{j=1}^{J} K_{j} \log \left[1-\frac{\lambda_{s}^{j}}{e_{0, j}}+\frac{\lambda_{s}^{j}}{e_{0, j}} \exp \left(s e_{0, j}\right)\right],
\end{aligned}
$$

$\mathrm{J}$ : the number of different traffic types,

$K_{j}$ : the number of connections that belong to traffic type $\mathrm{j}$,

$e_{0, j}$ : the lossless effective bandwidth for traffic type $\mathrm{j}$,

$\lambda_{s}^{j}$ : the sustainable cell rate for traffic type $\mathrm{j}$.

Under the boundary condition

$$
\sum_{j=1}^{J} K_{j} e_{j}=C
$$

the lossy effective bandwidth $e_{j}$ of traffic type $\mathrm{j}$ is given by:

$$
e_{j}=\frac{\log \left[1-\frac{\lambda_{s}^{j}}{e_{0, j}}+\frac{\lambda_{s}^{j}}{e_{0, j}} \exp \left(s e_{0, j}\right)\right]}{s^{*}+(\log L) / C} \quad(j=1,2, \cdots, J)
$$

where $s^{*}$ is where $F_{K}(s)$ is maximized and $L$ is CLR.

The statistical multiplexing gain $g$ compares lossy effective bandwidth with lossless effective bandwidth:

$$
g_{j}=e_{0, j} / e_{j} \quad(j=1,2, \cdots, J) .
$$

Intuitively, if $\mathrm{C}$ is large enough, there exists a statistical multiplexing gain; if $\mathrm{C}$ is small enough, there is no statistical multiplexing gain. Thus, there must exist a critical bandwidth $C_{c}$ that is the smallest quantity such that the statistical multiplexing gain $g$ exceeds unity for all $C>C_{c}$. Different traffic types may have different critical bandwidth. The critical bandwidth of traffic type $\mathrm{j}$ is given by

$$
C_{c, j}=\frac{e_{0, j} \log (1 / L)}{\log \left(e_{0, j} / \lambda_{s}^{j}\right)} \quad(j=1,2, \cdots, J)
$$

To illustrate $C_{c, j}$, we need to consider three cases for a two-type model:

1) $C<\min \left(C_{c, 1}, C_{c, 2}\right)$

According to the definition of $C_{c}$, it can be seen that both traffic types are NS-VBR (Nonstatisticallymultiplexible VBR), i.e., "bad" in the sense that the sources are too "large" with respect to the node bandwidth. 
2) $C>\max \left(C_{c, 1}, C_{c, 2}\right)$

According to the definition of $C_{c}$, both traffic types are S-VBR (Statistically-multiplexible VBR).

3) $C_{c, 1}<C<C_{c, 2}$

According to the definition of $C_{c}$, type 1 is S-VBR and type 2 is NS-VBR for the given node bandwidth (and, implicitly, node buffer).

\subsection{NEC's CAC}

To support multiple classes of services in an ATM switch, Ramamurthy and Ren proposed a multiclass CAC scheme [11]. In their CAC for VBR service, a two-phase algorithm was developed. Similar to Lucent's CAC, lossless multiplexing is considered in the first phase and statistical multiplexing is considered in the second phase.

The system model used in their CAC is a network node with the overall buffer allocated to VBR traffic $B_{v b r}$, the maximum bandwidth allocated to VBR traffic $C_{v b r}^{\max }$, and dual leaky-bucket regulated input traffic source. Assume that $\mathrm{n}$ VBR connections have been admitted, where the i-th connection has UPC parameters $\left(\lambda_{p}^{i}, \lambda_{s}^{i}, B_{s}^{i}\right), i=1,2, \cdots, n$. Let $C_{v b r}^{\text {old }}$ be the bandwidth currently allocated to the VBR class, supporting the $\mathrm{n}$ VBR connections.

\subsubsection{Lossless Multiplexing}

They first characterize the departure process of dual leaky buckets as the worst-case traffic model described in Section 2.1 with "on" and "off" periods given by

$$
T_{o n}^{*}=\frac{B_{s}^{*}}{\lambda_{p}^{*}} \quad \text { and } \quad T_{o f f}^{*}=\frac{B_{s}^{*}}{\lambda_{p}^{*}} \cdot \frac{\left(\lambda_{p}^{*}-\lambda_{s}^{*}\right)}{\lambda_{s}^{*}} .
$$

The probability such a source is in the "on" state is given by

$$
P_{o n}^{*}=\frac{\lambda_{s}^{*}}{\lambda_{p}^{*}}
$$

If a new connection with UPC parameters $\left(\lambda_{p}^{*}, \lambda_{s}^{*}, B_{s}^{*}\right)$ is requested, they derived the new overall bandwidth $C_{v b r}^{n e w}$ to be assigned to the VBR class with zero loss probability given by

$$
C_{v b r}^{n e w}=\max \left(\left(\lambda_{p}^{*}+\sum_{i=1}^{n} \lambda_{p}^{i}\right)\left(1-\frac{B_{v b r}}{B_{s}^{*}+\sum_{i=1}^{n} B_{s}^{i}}\right)^{+}, \lambda_{s}^{*}+\sum_{i=1}^{n} \lambda_{s}^{i}\right),
$$

where

$$
(x)^{+}=\max (x, 0)
$$

The additional bandwidth required to support the new connection is given by

$$
\delta_{1}=C_{v b r}^{n e w}-C_{v b r}^{o l d}
$$




\subsubsection{Statistical Multiplexing}

To extract statistical multiplexing gain, they used a modified source model. They defined a threshold by:

$$
T_{N}=\frac{B_{v b r}}{2 \cdot C_{v b r}^{\max }}
$$

They constructed a new two-state source model with high rate $\lambda_{H}^{*}$ and low rate $\lambda_{L}^{*}$ given by

$$
\begin{aligned}
& \lambda_{H}^{*}=\min \left(1, \frac{T_{o n}^{*}}{T_{N}}\right) \lambda_{p}^{*}+\max \left(0,1-\frac{T_{o n}^{*}}{T_{N}}\right) \lambda_{s}^{*} \\
& \lambda_{L}^{*}=\max \left(0,1-\frac{T_{o n}^{*}}{T_{N}}\right) \lambda_{s}^{*} .
\end{aligned}
$$

Corresponding to the on-off source model, the high-rate state and the low-rate state occur with probability $P_{o n}^{*}$ and $\left(1-P_{o n}^{*}\right)$. Thus, the modified source has the same average rate $\lambda_{s}^{*}$ as the original one.

Based on the modified source model and Gaussian approximation of the aggregate instantaneous rate of all VBR connections, they derived $C_{v b r}^{n e w}$ given by

$$
C_{v b r}^{n e w}=\mu_{n e w}+a \cdot \sigma_{n e w}
$$

where

$$
\begin{aligned}
& \mu_{\text {new }}=\mu_{\text {old }}+\lambda_{s}^{*} \\
& \sigma_{\text {new }}^{2}=\sigma_{\text {old }}^{2}+\left(\lambda_{H}^{*}-\lambda_{L}^{*}\right)^{2} P_{o n}^{*}\left(1-P_{\text {on }}^{*}\right) \\
& \eta=\frac{\mu_{\text {new }} \sqrt{2 \pi}}{\sigma_{\text {new }}} \cdot \varepsilon \\
& a \approx 1.8-0.46 \cdot \log _{10}(\eta)
\end{aligned}
$$

where $\varepsilon=C L R$ if only $C L R$ is considered. Therefore, the additional bandwidth required to support the new connection is given by

$$
\delta_{2}=C_{v b r}^{n e w}-C_{v b r}^{o l d}
$$

Thus, combining the lossless and lossy models, the additional required bandwidth $\Delta_{v b r}$ is given by

$$
\Delta_{v b r}=\min \left(\delta_{1}, \delta_{2}\right) .
$$




\section{Our CAC Schemes Based on Tagged Probability}

In many analyses of loss in ATM networks, overflow probability is used rather than Cell Loss Ratio (CLR), which is the fraction of lost cells among total arrival, because of its computational complexity. Our numerical investigations indicate that the loss-probability-to-overflow-probability ratio tends to decrease as the number of sources increases. Therefore, an overflow-based QoS guarantee may be very conservative for a large capacity system compared with a loss-based one. Futhermore, the overflow probability is not useful in some priority shcemes where both high and low priority traffic completely share a common buffer [17]. Song and Boorstyn proposed an efficient way to approximate the upper bound on loss probability called tagged probability [17]. The tagged probability is a very tight upper bound on loss, and can be estimated by using sophisticated matrix theory and Gaussian approximation.

\subsection{Loss Probability, Tagged Probability, and Overflow Probability}

To explain why our CAC algorithms can achieve higher resource utilization, we first discuss the difference between loss probability and overflow probability. By definition, the exact loss probability, $L$, in a finite buffer of size B (see Figure 2(a)) can be given by

$$
L=\frac{E\left[(\lambda-c)^{+} \mid X=B\right]}{\bar{\lambda}} P_{F B}(X=B),
$$

where

$(x)^{+}=\max (x, 0)$,

$\bar{\lambda}$ : the average arrival rate of all the sources,

$\lambda$ : the aggregate instantaneous rate,

$c$ : link capacity or allocated bandwidth,

$X$ : buffer occupancy,

$P_{F B}$ : the probability in finite buffer model.

Since the exact loss probability, $L$, is not readily obtained, people tend to use the overflow probability, $P_{I B}(X>B)$, in their CAC algorithms, which is the probability that the buffer occupancy is above threshold $B$ in an infinite buffer model (see Figure 2(b)). In most cases, the overflow probability, $P_{I B}(X>B)$, is greater than the loss probability, $L$, although there are extreme cases where $P_{I B}(X>B)<L$. Thus, $P_{I B}(X>B)$ is usually used as an upper bound on $L$. To tighten the upper bound on $L$, Song and Boorstyn introduced the tagged probability. Consider an infinite buffer model with a threshold set at B. When the buffer occupancy is above the threshold B, we tag the incoming traffic arriving at a rate in excess of c. Define the tagged probability as $T g$. Then $T g$ is expressed by

$$
T g=\frac{E\left[(\lambda-c)^{+} \mid X>B\right]}{\bar{\lambda}} P_{I B}(X>B) .
$$

We observe that the overflow probability, $P_{I B}(X>B)$, is greater than the probability $P_{F B}(X=$ $B$ ) (see Figure 2(c)). Comparing Equation (34) with Equation (33), we can obtain the property 


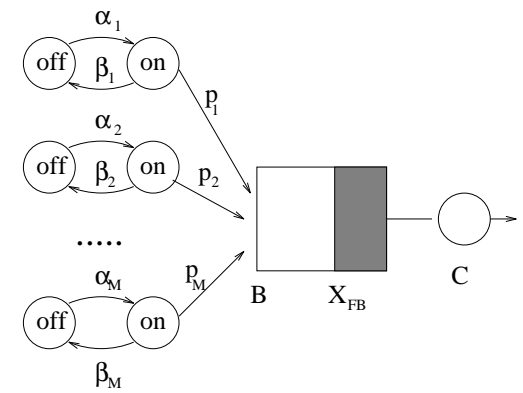

(a) Finite Buffer Model

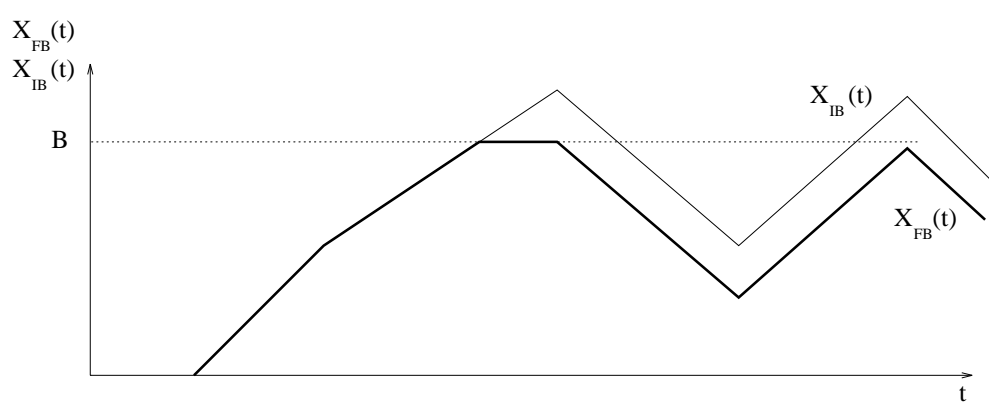

(c) Buffer occupancy variation in the different models.

Figure 2: Buffer occupancy comparison between finite buffer and infinite buffer models.

that the tagged probability, $T g$, is greater than the loss probability, $L$, i.e., CLR. Therefore, $T g$ can be used as an upper bound on CLR.

Define

$$
\begin{aligned}
& K=\frac{E\left[(\lambda-c)^{+} \mid X>B\right]}{\bar{\lambda}}, \\
& P_{o v}=P_{I B}(X>B) .
\end{aligned}
$$

So we have

$$
C L R \leq T g=K \cdot P_{o v}
$$

Thus, we can regard $K$ as the loss-probability-to-overflow-probability ratio by using

$$
C L R \approx K \cdot P_{o v}
$$

Using the Kronecker product property of the dominant eigenvector of the system equation and Gaussian approximation, Song and Boorstyn derived an efficient estimation of $K$ given by [17]:

$$
K=\frac{\frac{\sigma}{\sqrt{2 \pi}} e^{-\frac{(c-\mu)^{2}}{2 \sigma^{2}}}+(\mu-c)^{+}}{\bar{\lambda}}
$$

where

$$
\mu=\sum_{i} \mu_{i}
$$




$$
\sigma^{2}=\sum_{i} \sigma_{i}^{2}
$$

where $\mu_{i}$ is the average rate of individual sources and $\sigma_{i}$ is the standard deviation of the rate of individual sources. Although people assume $K=1$, i.e., $C L R \approx P_{o v}$, our numerical investigations have shown that $K$ is usually less than unity, i.e., usually in the range of $0.001 \sim 0.1$. Thus, the introduction of $K$ makes our CAC algorithms achieve higher resource utilization. In the following subsections, we describe our three modified CAC algorithms, which are based on NEC's CAC, Effective Bandwidth, and Lucent's CAC, respectively.

\subsection{TAP Algorithm}

The TAP algorithm is so called since it is derived from TAgged Probability and it taps the resources to achieve higher utilization. The TAP algorithm is based on NEC's CAC. Assume we have the same model and parameters as those in Section 2.4.2. From the discussion in Section 3.1, we have

$$
\varepsilon=P_{o v}=\frac{C L R}{K}
$$

Different from Section 2.4.2, we define a new threshold $T_{N}$ and a parameter $k_{N}$ :

$$
\begin{aligned}
& T_{N}=s \cdot \frac{B_{v b r}}{C_{v b r}^{\max }}, \\
& k_{N}=\frac{T_{o n}^{*}}{T_{N}} .
\end{aligned}
$$

To investigate the effectiveness and sensitivity of NEC's modified source model in Section 2.4.2, we construct our source model by introducing factor $s$. The basic time interval is proportional to the buffer-bandwidth ratio. When $s=1, T_{N}$ is the longest time that a packet in a First-in-First-Out (FIFO) queue will wait when the buffer is full. We define the effective buffer $b_{E}$

$$
b_{E}=\frac{B_{s}}{s} .
$$

So $s$ is the ratio between the maximum burst size $B_{s}$ and the effective buffer. We observe that increasing ratio $s$ can increase the system utilization, as Section 4 discusses.

Similar to Section 2.4.2, we construct a new two-state source model with high rate $\lambda_{H}^{*}$ and low rate $\lambda_{L}^{*}$ given by

$$
\begin{aligned}
& \lambda_{H}^{*}=\left\{\begin{array}{cc}
k_{N} \lambda_{p}^{*}+\left(1-k_{N}\right) \lambda_{s}^{*} & \text { if } k_{N}<1 \text { or } \frac{B_{s}^{*}}{s \cdot B_{v b r}}<\frac{\lambda_{p}}{C_{v b r}^{\text {max }}} \\
\lambda_{p}^{*} & \text { if } k_{N} \geq 1 \text { or } \frac{B_{s}^{*}}{s \cdot B_{v b r}} \geq \frac{\lambda_{p}}{C_{v b r}^{m a x}}
\end{array},\right. \\
& \lambda_{L}^{*}=\left\{\begin{array}{cc}
\left(1-k_{N}\right) \lambda_{s}^{*} & \text { if } k_{N}<1 \text { or } \frac{B_{s}^{*}}{s \cdot B_{v b r}}<\frac{\lambda_{p}}{C_{v b r}^{\text {max }}} \\
0 & \text { if } k_{N} \geq 1 \text { or } \frac{B_{s}^{*}}{s \cdot B_{v b r}} \geq \frac{\lambda_{p}}{C_{v b r}^{\max }}
\end{array} .\right.
\end{aligned}
$$


Thus, sources with busy periods that are small compared to the basic time scale, are modeled by their weighted averages with parameter $k_{N}$. Sources whose busy periods are longer than the basic time interval $T_{N}$ are still modeled as the worst-case on-off model. This step will reduce the rate variance and thus the bandwidth requirement for sources with short busy periods. When $s=\frac{1}{2}$, the TAP algorithm becomes NEC's CAC. In the following, we assume $s=\frac{1}{2}$ for the sake of comparison. We call the estimated bandwidth obtained by Equations (20) and (22) lossless equivalent bandwidth, and call the estimated bandwidth obtained by Equations (26) and (31) lossy equivalent bandwidth.

Proposition 1: Define

$$
K(c)=\frac{\frac{\sigma}{\sqrt{2 \pi}} e^{-\frac{(c-\mu)^{2}}{2 \sigma^{2}}}+(\mu-c)^{+}}{\bar{\lambda}} .
$$

$K(c)$ is monotonic, decreasing with increasing $c$ when $c \geq \mu$.

Proof: Suppose $\mu<c_{1}<c_{2}$. Then

$$
\mu-c_{2}<\mu-c_{1}<0
$$

Since $(x)^{+}=\max (x, 0)$, then

$$
\left(\mu-c_{2}\right)^{+}=\left(\mu-c_{1}\right)^{+}=0 .
$$

Take the square of (49)

$$
\left(\mu-c_{2}\right)^{2}>\left(\mu-c_{1}\right)^{2}
$$

Take negative

$$
-\left(\mu-c_{2}\right)^{2}<-\left(\mu-c_{1}\right)^{2} .
$$

Since $\sigma>0$ and $\bar{\lambda}>0$, then

$$
\frac{\frac{\sigma}{\sqrt{2 \pi}} e^{-\frac{\left(c_{2}-\mu\right)^{2}}{2 \sigma^{2}}}}{\bar{\lambda}}<\frac{\frac{\sigma}{\sqrt{2 \pi}} e^{-\frac{\left(c_{1}-\mu\right)^{2}}{2 \sigma^{2}}}}{\bar{\lambda}} .
$$

From (45) and (48)

$$
K\left(c_{1}\right)>K\left(c_{2}\right) \text {. }
$$

Corollary 1: Given $\mu<C_{v b r}^{\text {old }}<C_{v b r}^{n e w}, K\left(C_{v b r}^{\text {old }}\right)>K\left(C_{v b r}^{n e w}\right)$.

Proposition 2: Define

$$
\begin{aligned}
& g(a)=\mu+a \cdot \sigma \\
& a(\eta)=1.8-0.46 \cdot \log _{10}(\eta) \\
& \eta(\varepsilon)=\frac{\mu \sqrt{2 \pi}}{\sigma} \cdot \varepsilon \\
& \varepsilon(K)=\frac{C L R}{K} .
\end{aligned}
$$


$g(K)$ is monotonic, increasing with increasing $K(K>0)$.

Proof: Suppose $0<K_{1}<K_{2}$. Then

$$
\frac{1}{K_{2}}<\frac{1}{K_{1}} .
$$

For $C L R>0$

$$
\frac{C L R}{K_{2}}<\frac{C L R}{K_{1}}
$$

That is

$$
\varepsilon\left(K_{2}\right)<\varepsilon\left(K_{1}\right) .
$$

Since $\mu>0$ and $\sigma>0$, from (56)

$$
\eta\left(K_{2}\right)<\eta\left(K_{1}\right) .
$$

Then, from (55)

$$
a\left(K_{2}\right)>a\left(K_{1}\right) .
$$

Thus, from (54)

$$
g\left(K_{2}\right)>g\left(K_{1}\right) .
$$

When a new connection with the UPC parameters $\left(\lambda_{p}^{*}, \lambda_{s}^{*}, B_{s}^{*}\right)$ is requested, its CLR requirement can be approximated by

$$
C L R \approx K \cdot P_{o v}=K \cdot \varepsilon .
$$

To estimate $K$ through Equation (39), we have to know $\mu, \sigma, c$. The standard variation $\sigma$, i.e., $\sigma_{\text {new }}$, is given by

$$
\sigma_{\text {new }}^{2}=\sigma_{\text {old }}^{2}+\lambda_{s}^{*}\left(\lambda_{p}^{*}-\lambda_{s}^{*}\right)
$$

Since the total required bandwidth $c$, i.e., $C_{v b r}^{n e w}$, is to be estimated and unknown, we have to find an approximation that should be conservative. From Corollary 1 , we know $K\left(C_{v b r}^{\text {old }}\right)$ is an upper bound of $K\left(C_{v b r}^{n e w}\right)$ when $\mu<C_{v b r}^{\text {old }}<C_{v b r}^{n e w}$. Furthermore, Proposition 2 tells us $g\left(C_{v b r}^{\text {old }}\right)>g\left(C_{v b r}^{n e w}\right)$. So $g\left(C_{v b r}^{o l d}\right)$ is conservative. Therefore, $C_{v b r}^{\text {old }}$ can replace $c$ in Equation (39). To guarantee $C_{v b r}^{o l d}>\mu$, the mean $\mu$ has to be $\mu_{\text {old }}$ rather than $\mu_{\text {new }}$ since $C_{v b r}^{\text {old }}$ may be less than $\mu_{n e w}$ but must be greater than $\mu_{\text {old }}$ for stability. Thus we obtain $K$ by

$$
K=\frac{\frac{\sigma_{n e w}}{\sqrt{2 \pi}} e^{-\frac{\left(C_{v b r}^{o l d}-\mu_{o l d}\right)^{2}}{2 \sigma_{\text {new }}^{2}}}+\left(\mu_{\text {old }}-C_{v b r}^{\text {old }}\right)^{+}}{\mu_{\text {old }}},
$$

where $C_{v b r}^{\text {old }}$ is the sum of the effective bandwidth of the existing connections and $\mu_{\text {old }}$ is the sum of $\lambda_{s}$.

After obtaining $K$, we can calculate $\varepsilon$ using Equation (42). Then we can compute $C_{v b r}^{n e w}$ through (27-30) and (26). Finally, the additional required bandwidth for the new connection can be obtained through Equations (31) and (32). 
Proposition 3: TAP achieves higher utilization than NEC's CAC when $0<K<1$.

Proof: Since $0<K<1$, from Proposition 2:

$$
g(K)<g(1)
$$

where $g(K)$ is the estimated bandwidth by TAP and $g(1)$ is the estimated bandwidth by NEC's CAC. This means TAP can admit more connections with the same link capacity and thus achieves higher utilization than NEC's CAC.

Since TAP is based on the tagged probability, which is an upper bound on CLR, TAP still keeps conservativeness without violating the QoS of the connections. The conservativeness and Proposition 3 will be further verified by our simulation and numerical results.

\subsection{Modified Effective Bandwidth (MEB)}

Assume we have the same model and parameters as those in Section 2.2. From the discussion in Section 3.1, we have

$$
p=P_{o v}=\frac{C L R}{K} .
$$

Then $\eta$ can be given by

$$
\eta=\frac{\log P_{o v}}{B}=\frac{\log (C L R / K)}{B} .
$$

Then, the effective bandwidth of the source, e, is also given by Equation (5).

Fact 1: The maximum real eigenvalue $e(\eta)$ of the essentially nonnegative matrix $\Lambda(\eta)=$ $\left[\Lambda-\frac{1}{\eta} M\right]$ is monotonic, decreasing with increasing $\eta$,

$$
e^{\prime}(\eta)<0 \quad(\eta<0)
$$

Proposition 4: $\eta(K)$ is monotonic, decreasing with increasing $K(K>0)$.

Proof: Suppose $0<K_{1}<K_{2}$. Then

$$
\frac{1}{K_{2}}<\frac{1}{K_{1}}
$$

For $C L R>0$ and $B>0$

$$
\frac{\log \left(C L R / K_{2}\right)}{B}<\frac{\log \left(C L R / K_{1}\right)}{B} .
$$

That is $\eta\left(K_{2}\right)<\eta\left(K_{1}\right)$.

Proposition 5: The effective bandwidth $e(K)$ is monotonic, increasing with increasing $K$ $(K>0)$.

Proof: From Fact 1 and Proposition 4, it can be seen that $e(K)$ is monotonic, increasing with increasing $K$.

When a new connection with UPC parameters $\left(\lambda_{p}^{*}, \lambda_{s}^{*}, B_{s}^{*}\right)$ is requested, its CLR requirement can be approximated by

$$
C L R \approx K \cdot P_{o v} .
$$


To estimate $K$ through Equation (39), we have to know $\mu, \sigma, c$. The standard variation $\sigma$, i.e., $\sigma_{n e w}$, is given by

$$
\sigma_{\text {new }}^{2}=\sigma_{\text {old }}^{2}+\lambda_{s}^{*}\left(\lambda_{p}^{*}-\lambda_{s}^{*}\right)
$$

Since the total required bandwidth $c$, i.e., $C_{v b r}^{n e w}$, is to be estimated and unknown, we have to find an approximation that should be conservative. From Corollary 1, we know $K\left(C_{v b r}^{\text {old }}\right)$ is an upper bound of $K\left(C_{v b r}^{n e w}\right)$ when $\mu<C_{v b r}^{\text {old }}<C_{v b r}^{n e w}$. Furthermore, Proposition 5 tells us $e\left(C_{v b r}^{\text {old }}\right)>e\left(C_{v b r}^{n e w}\right)$. So $e\left(C_{v b r}^{\text {old }}\right)$ is conservative. Therefore, $C_{v b r}^{\text {old }}$ can replace $c$ in Equation (72). To guarantee $C_{v b r}^{\text {old }}>\mu$, the mean $\mu$ has to be $\mu_{\text {old }}$ rather than $\mu_{\text {new }}$ since $C_{v b r}^{\text {old }}$ may be less than $\mu_{n e w}$ but must be greater than $\mu_{\text {old }}$ for stability. Thus we can also obtain $K$ through Equation (65).

After obtaining $K$, we can calculate $\eta$ using Equation (68). Finally, the effective bandwidth of the new connection can be obtained through Equation (5).

Proposition 6: MEB achieves higher utilization than the CAC based on Effective Bandwidth when $0<K<1$.

Proof: Since $0<K<1$, from Proposition 5:

$$
e(K)<e(1)
$$

where $e(K)$ is the estimated bandwidth by MEB and $e(1)$ is the estimated bandwidth by the CAC based on Effective Bandwidth. This means MEB can admit more connections with the same link capacity and thus achieves higher utilization than the CAC based on Effective Bandwidth.

Since MEB is based on the tagged probability, which is an upper bound on Cell Loss Ratio, MEB still keeps conservativeness without violating the QoS of the connections. The conservativeness and Proposition 6 will be further verified by our simulation and numerical results.

\subsection{Modified Statistical Multiplexing (MSM) Algorithm}

MSM is based on Lucent's CAC. Assume we have the same model and parameters as those in Section 2.3.2. From the discussion in Section 3.1, we have

$$
L=P_{o v}=\frac{C L R}{K} \text {. }
$$

Proposition 7: Define

$$
\begin{aligned}
& e_{j}(L)=\frac{\log \left[1-\frac{\lambda_{s}^{j}}{e_{0, j}}+\frac{\lambda_{s}^{j}}{e_{0, j}} \exp \left(s e_{0, j}\right)\right]}{s^{*}+(\log L) / C} \quad(j=1,2, \cdots, J) \\
& L(K)=\frac{C L R}{K} \\
& K(c)=\frac{\frac{\sigma}{\sqrt{2 \pi}} e^{-\frac{(c-\mu)^{2}}{2 \sigma^{2}}}+(\mu-c)^{+}}{\bar{\lambda}} .
\end{aligned}
$$

$e_{j}(K)$ is monotonic, increasing with increasing $K(K>0)$. 
The proof of Proposition 7 is similar to Proposition 2. From Corollary 1, we know $K\left(C_{v b r}^{o l d}\right)$ is an upper bound of $K\left(C_{v b r}^{n e w}\right)$ when $\mu<C_{v b r}^{\text {old }}<C_{v b r}^{\text {new }}$. Furthermore, Proposition 7 tells us $e_{j}\left(C_{v b r}^{\text {old }}\right)>e_{j}\left(C_{v b r}^{n e w}\right)$. So $e_{j}\left(C_{v b r}^{\text {old }}\right)$ is a conservative estimation. Thus, we can use Equation (65) to obtain $K$ as well. Then the lossy effective bandwidth $e_{j}$ of the traffic type $j$ can be obtained by solving Equations (12) and (13).

Proposition 8: MSM achieves higher utilization than Lucent's CAC when $0<K<1$.

Proof: Since $0<K<1$, from Proposition 7:

$$
e_{j}(K)<e_{j}(1)
$$

where $e_{j}(K)$ is the estimated bandwidth by MSM and $e_{j}(1)$ is the estimated bandwidth by Lucent's CAC. This means MSM can admit more connections with the same link capacity and thus achieves higher utilization than Lucent's CAC.

Since MSM is based on the tagged probability, which is an upper bound on Cell Loss Ratio, MSM still keeps conservativeness without violating the QoS of the connections. The conservativeness and Proposition 8 will be further verified by our simulation and numerical results.

\section{Simulation and Numerical Investigations}

Many CAC schemes have been evaluated only with exponential on-off sources that have properties quite different from those observed by measurement studies of many actual traffic streams [1820]. Knightly showed that CAC schemes that work demonstrably well with exponential on-off sources can suffer from considerable inaccuracies when applied to multiple time scale sources such as compressed VBR video [21]. Thus, to simulate the admission control under realistic traffic, we use two traces of bursty MPEG (Motion Picture Expert Group) video. We determine the admissible regions of the $\mathrm{CAC}$ schemes of interest for the traces through numerical methods. Our numerical investigations are based on the UPC parameters of the video traces that are obtained by Mark and Ramamurthy's estimation procedure [22]. Since there is no exact analysis for the traces, we use the trace-driven simulations as criteria to evaluate the utilization of different CAC schemes given the same UPC parameters of the sources.

In the example of Table 1, we use an MPEG-1 coded sequence called Mobi trace. This empirical data sequence has a peak cell rate of $17.38 \mathrm{Mbps}$ and an average cell rate of $8.58 \mathrm{Mbps}$. Using the method developed in [22], we can choose UPC parameters $\left(\lambda_{p}=17.38 \mathrm{Mbps}, \lambda_{s}=11.87 \mathrm{Mbps}\right.$, $B_{s}=83$ cells) to characterize the Mobi trace. We consider an ATM multiplexer with a buffer size of 500 cells and a QoS constraint: CLR $<10^{-5}$. In Table 1, given the above UPC parameters for the Mobi trace and the different link capacities (i.e., $C_{v b r}=45 \mathrm{Mbps}, 100 \mathrm{Mbps}, 150 \mathrm{Mbps}$, and $300 \mathrm{Mbps}$ ), we show the maximum number of Mobi traces, which can be admitted without violating the CLR requirements, for various schemes and simulations. The Equivalent Bandwidth is based on [4]. The PNNI (Private Network-Network Node) CAC is the generic CAC proposed by the ATM Forum [6]. Table 1 shows that TAP and NEC's CAC have the same performance for Mobi traces. This is due to the fact that TAP and NEC's CAC use the same algorithm for lossless 
multiplexing described in Section 2.4.1, and only the lossless equivalent bandwidth is considered since the lossless equivalent bandwidth is less than the lossy equivalent bandwidth for Mobi traces. Table 1 also shows that Lucent's CAC has the same performance as that of NEC's CAC. This is because both of them use similar assumptions in lossless multiplexing: the ratio between the buffer and the bandwidth allocated to the individual connections is proportional to the ratio between the buffer size and the bandwidth capacity allocated for total VBR traffic.

\begin{tabular}{|c|c|c|c|c|}
\hline & \multicolumn{4}{|c|}{ Link Capacity (Buffer=500 cells) } \\
\hline CAC mechanisms & $45 \mathrm{Mbps}$ & $100 \mathrm{Mbps}$ & $150 \mathrm{Mbps}$ & $300 \mathrm{Mbps}$ \\
\hline Simulation & 4 & 9 & 14 & 28 \\
\hline NEC's CAC & 3 & 8 & 12 & 23 \\
\hline TAP & 3 & 8 & 12 & 23 \\
\hline Effective Bandwidth & 3 & 7 & 10 & 21 \\
\hline MEB & 3 & 7 & 11 & 22 \\
\hline Lucent's CAC & 3 & 8 & 12 & 23 \\
\hline MSM & 3 & 8 & 12 & 23 \\
\hline Equivalent Bandwidth & 3 & 7 & 10 & 21 \\
\hline PNNI & 2 & 5 & 8 & 17 \\
\hline Peak Rate & 2 & 5 & 8 & 17 \\
\hline
\end{tabular}

*The numbers shown in the table are the number of MPEG-1 Mobi trace connections that can be admitted by various CAC schemes.

Table 1: Performance comparison for various CAC schemes using Mobi traces

In the example of Table 2, we use a more bursty MPEG-1 coded trace called Flower Garden. This trace has a peak cell rate of $13.91 \mathrm{Mbps}$ and an average cell rate of $3.1 \mathrm{Mbps}$. Based on [22], the UPC parameters we choose for this trace is $\left(\lambda_{p}=13.91 \mathrm{Mbps}, \lambda_{s}=3.6 \mathrm{Mbps}, B_{s}=1479\right.$ cells). The QoS constraint is: CLR $<10^{-5}$. In Table 2, given the above UPC parameters for the Flower Garden trace and the different link capacities (i.e., $C_{v b r}=45 \mathrm{Mbps}, 100 \mathrm{Mbps}, 150 \mathrm{Mbps}$, and 300Mbps), we show the maximum number of Flower Garden traces, which can be admitted without violating the CLR requirements, for various schemes and simulations. Table 2 shows that TAP is more efficient than any of the other CAC schemes and MSM has comparable performance as that of TAP. We observe that the larger the link capacity is, the more statistical multiplexing gain TAP achieves. It is noteworthy that Lucent's CAC, which is based on Chernoff bound, has the same performance as that of Equivalent Bandwidth, which is based on Gaussian approximation and Effective Bandwidth. Our MEB is more efficient than Lucent's CAC and Equivalent Bandwidth when the link capacity is large. We notice that even TAP is still very conservative. This is because TAP still uses an on-off model $\left(\lambda_{p}^{*}, 0\right)$ when $T_{o n}^{*}>T_{N}$, i.e., $\frac{B_{s}^{*}}{B_{v b r} / 2}>\frac{\lambda_{p}^{*}}{C_{v b r}}$. Frames with a large size like $B_{s}$ may be very rare. Thus, without more information such as the distribution of the rate or frame size, UPC-parameter-based CAC schemes can only choose a conservative estimation. We observe that the larger the link capacity is, the more statistical multiplexing gain there is. Thus, 
$T_{N}$ should change as the link capacity changes. This is the very reason why we introduce ratio $s$ in our modified source model described in Section 3.2.

\begin{tabular}{|c|c|c|c|c|}
\hline & \multicolumn{4}{|c|}{ Link Capacity (Buffer=500 cells) } \\
\hline CAC mechanisms & $45 \mathrm{Mbps}$ & $100 \mathrm{Mbps}$ & $150 \mathrm{Mbps}$ & $300 \mathrm{Mbps}$ \\
\hline Simulation & 8 & 17 & 30 & 77 \\
\hline NEC's CAC & 3 & 8 & 16 & 42 \\
\hline TAP & 3 & 11 & 20 & 52 \\
\hline Effective Bandwidth & 3 & 7 & 11 & 22 \\
\hline MEB & 3 & 7 & 13 & 37 \\
\hline Lucent's CAC & 3 & 7 & 13 & 26 \\
\hline MSM & 3 & 11 & 20 & 50 \\
\hline Equivalent Bandwidth & 3 & 7 & 13 & 36 \\
\hline PNNI & 3 & 7 & 13 & 36 \\
\hline Peak Rate & 3 & 7 & 13 & 36 \\
\hline
\end{tabular}

*The numbers shown in the table are the number of MPEG-1 Flower Garden trace connections that can be admitted by various CAC schemes.

Table 2: Performance comparison for various CAC schemes using Flower Garden traces

Figure 3 shows that the loss-probability-to-overflow-probability ratio $K$ decreases when the number of Mobi sources increases. This figure also indicates that $K$ is within the range of 0.01 to 0.5 when the number of Mobi sources is greater than two. Figure 4 shows that the loss-probabilityto-overflow-probability ratio $K$ decreases when the number of Flower Garden sources increases. This figure also indicates that $K$ is within the range of 0.001 to 0.5 when the number of Flower Garden sources is greater than two. Thus the loss-probability-to-overflow-probability ratio $K$ tends to decrease as the number of sources increases.

Figures 5 and 6 show that by increasing $s$ from $\frac{1}{2}$ to 170, TAP and NEC's CAC can admit more connections. So increasing $s$ opens another way to increase system utilization. But it may also lead to under-estimating the required bandwidth. We observe that there is a critical value $s^{*}$ for certain link capacity, which makes TAP or NEC's CAC achieve the highest possible utilization with guaranteed QoS. It is an engineering problem to determine which $s^{*}$ is appropriate to achieve the highest possible utilization while still guaranteeing the QoS for the extremal traffic. To be more specific, since every type of traffic has a critical value $s^{*}$, we choose the smallest $s^{*}$, which corresponds to the extremal traffic. Since Lucent's CAC uses predefined types, there must exist an extremal type among them. We can find $s_{j}^{*}$ for this extremal type and use it as system critical value $s^{*}$. Thus, TAP or NEC's CAC can achieve the highest possible utilization with guaranteed QoS. Figures 5 and 6 also raise a question: since introducing $s^{*}$ is enough to increase the utilization while guaranteeing QoS, do we still need to calculate the loss-probability-to-overflow-probability ratio $K$ ? The answer is that if $s^{*}$ is not readily available, $K$ is still needed to increase the utilization.

We notice that the lower bound of lossless equivalent bandwidth of a source is the sustainable cell 
rate, $\lambda_{s}$, of the source. This is because Equation (20) indicates that lossless equivalent bandwidth must be greater than or equal to $\lambda_{s}$. We also notice that the lower bound of lossy equivalent bandwidth of a source is the sustainable cell rate, $\lambda_{s}$, of the source. It can be seen from Equations (43) and (44) that when $s \rightarrow \infty, k_{N} \rightarrow 0$. Thus, from Equations (46) and (47), we have $\lambda_{H}^{*} \rightarrow \lambda_{s}^{*}$ and $\lambda_{L}^{*} \rightarrow \lambda_{s}^{*}$, which means the source model becomes a Constant Bit Rate (CBR) source with $\lambda_{p}=\lambda_{s}=\lambda_{s}^{*}$. Therefore, the lower bound of lossy equivalent bandwidth of a source is the sustainable cell rate, $\lambda_{s}$, of the source. So by increasing $s$, the maximum number of identical connections that can possibly be admitted is the largest integer that is less than $C_{v b r} / \lambda_{s}$. It is worth mentioning that another way to increase utilization is measurement-based admission control [23].

Figure 7 shows that a large buffer can increase the number of admissible connections for Mobi traces. This suggests that Mobi sources (which are NS-VBR) can benefit from a large buffer size. Figure 8 shows that the buffer size has no effect on the number of admissible connections for Flower Garden traces. This suggests that Flower Garden sources (which are S-VBR) cannot gain anything from a large buffer size. Figures 7 and 8 also shed some light on what traffic types shared-memory ATM switches may have an effect on.

From Figure 9, we have the following observations:

1) Effective Bandwidth has the smallest admissible region.

2) MEB and Lucent's CAC have comparable performances. When the number of Flower Garden connections is greater than 17, MEB performs a little better than Lucent's CAC. This suggests that MEB performs a little better than Lucent's CAC when the number of S-VBR sources is large.

3) NEC's CAC performs better than Effective Bandwidth when the number of Flower Garden connections is greater than 11. It also performs better than Lucent's CAC when the number of Flower Garden connections is greater than 20 and better than MEB when the number of Flower Garden connections is greater than 23. This suggests that NEC's CAC performs better than MEB, Lucent's CAC, and Effective Bandwidth when the number of S-VBR sources is large. This is because NEC's CAC is based on Gaussian approximation and Gaussian approximation assumes a large number of connections, i.e., S-VBR sources in this example.

4) TAP even performs worse than Effective Bandwidth when the number of Flower Garden connections is within the range of 2 to 6 . NEC's CAC performs worse than Effective Bandwidth when the number of Flower Garden connections is within the range of 2 to 10 . This is simply because there is no statistical multiplexing gain for TAP or NEC's CAC when the number of Flower Garden connections, i.e., S-VBR sources, is small.

5) TAP performs better than NEC's CAC, MEB, Lucent's CAC, and Effective Bandwidth when the number of Flower Garden connections is greater than 9.

6) MSM always performs better than NEC's CAC, MEB, Lucent's CAC, and Effective Bandwidth. It almost always performs better than TAP when the number of Flower Garden connections is within the range of 1 to 49. But when there are only Flower Garden connections, the maximum number of connections that MSM can admit, i.e., 50, is 2 less than the one admitted by TAP, i.e., 
52.

To evaluate the performance of various schemes under a very low CLR requirement, i.e., CLR $<$

$10^{-9}$, we performed computations under the same environment as that in Figure 9. The results shown in Figure 10 are similar to those shown in Figure 9, which corroborates the effectiveness of the proposed TAP algorithm under various QoS requirements.

Although it has been shown that MSM has the largest admissible region, MSM introduces the highest complexity (i.e., using both large deviation approximation and Gaussian approximation). Therefore, we do not recommend MSM algorithm. Instead, we recommend the TAP algorithm for CAC on high-speed ATM switches due to the simplicity and efficiency of the TAP algorithm.

\section{Conclusion}

The main objective of our research is to address the practical aspects of implementing a CAC on high-speed ATM switches. This motivates us to investigate the CAC schemes based on UPC parameters, which are standardized by the ATM Forum.

In this paper, we have developed three CAC algorithms and compared their performances with the CAC schemes in the literature. We have also discussed the worse-case traffic model since our CAC schemes are based on this model.

Our numerical investigations indicate that the loss-probability-to-overflow-probability ratio tends to decrease as the number of sources increases. Thus, the introduction of the loss-probability-tooverflow-probability ratio $K$ opens a new way to increase resource utilization while still guaranteeing QoS. Based on ratio $K$, we propose three CAC algorithms: TAP (TAgged Probability), MEB (Modified Effective Bandwidth), and MSM (Modified Statistical Multiplexing), which use the declared UPC parameters from traffic sources. Through analysis, simulation, and numerical investigation, we find that

- Our TAP algorithm is simple and performs well for homogeneous sources and heterogeneous sources under different buffer sizes and link capacities.

- Our MEB algorithm performs better than the Effective Bandwidth method.

- Among all the CAC schemes under investigation, MSM has the largest admissible region. This is because MSM uses both large deviation approximation and Gaussian approximation. Thus, MSM also has the highest complexity.

- The utilization can be further increased for TAP and NEC's CAC when $s$ (the ratio between the maximum burst size $B_{s}$ and the effective buffer) is introduced. How to find the critical value $s^{*}$ for a certain link capacity is an engineering problem. We suggest a method to obtain $s^{*}$ in the paper.

Due to the simplicity and efficiency of the TAP algorithm, we recommend the proposed TAP algorithm for CAC on high-speed ATM switches. 


\section{Acknowledgments}

The authors would like to thank Prof. Zhi-Li Zhang, Qiang Ren, Jongtae Song, and George Lapiotis for many fruitful discussions related to this work.

\section{References}

[1] ATM Forum, "User-network interface specification version 3.0," 1993.

[2] J. Y. Hui, "Resource allocation for broadband networks," IEEE Journal of Selected Areas in Communications, Vol. 6, No. 9, pp. 1598-1608, Dec. 1988.

[3] A. Elwalid and D. Mitra, "Effective bandwidth of general markovian traffic sources and admission control of high speed Networks," IEEE/ACM Trans. on Networking, Vol. 1, No. 3, pp. 329-343, June 1993.

[4] R. Guerin, H. Ahmadi, and M. Naghshineh, "Equivalent capacity and its application to bandwidth allocation in high-speed networks," IEEE Journal of Selected Areas in Communications, Vol. 9, No. 7, pp. 968-981, Sept. 1991.

[5] J. Suh, "Call admission and source characterization in high speed networks," Ph.D. Dissertation, Dept. of Electrical Engineering, Polytechnic University, Dec. 1995.

[6] ATM Forum, "Private network-network interface specification version 1.0 (PNNI 1.0)," 1996.

[7] A. Elwalid, D. Mitra, and R. Wentworth, "A new approach for allocating buffers and bandwidth to heterogeneous, regulated traffic in an ATM node," IEEE Journal of Selected Areas in Communications, Vol. 13, No.6, pp. 1115-1127, Aug. 1995.

[8] L. He and A. Wong, "Connection admission control design for Globeview-2000 ATM core switches," Bell Labs Technical Journal, pp. 94-111, January-March 1998.

[9] F. Lo Presti, Z-L Zhang, J. Kurose, D. Towsley, "Source time scale and optimal buffer/bandwidth trade-off for regulated traffic in an ATM node," IEEE Infocom'97, pp. 676683, March 1997.

[10] B. Jamoussi, S. Rabie, and O. Aboul-Magd, "Performance evaluation of connection admission control techniques in ATM networks," IEEE Globecom'96, pp. 659-664, Nov. 1996.

[11] G. Ramamurthy and Qiang Ren, "Multi-class connection admission control policy for high speed ATM switches," IEEE Infocom'97, pp. 965-974, March 1997.

[12] B. T. Doshi, "Deterministic rule based traffic descriptors for broadband ISDN: worst case behavior and connection acceptance control," IEEE Globecom'93, pp. 1759-1764, Nov. 1993. 
[13] D. Mitra and J. A. Morrison, "Independent regulated processes to a shared unbuffered resource which maximize the loss probability," unpublished manuscript, 1994.

[14] T. Worster, "Modeling deterministic queues: the leaky bucket as an arrival process," Proc. ITC-14, J. Labetoulle and J. W. Roberts, Eds. New York: Elsevier, 1994, pp. 581-590.

[15] N. Yamanaka, Y. Sato, and K. I. Sato, "Performance limitations of leaky bucket algorithm for usage parameter control of bandwidth allocation methods," IEICE Trans. Commun., Vol. E75-B, No. 2, 1992, pp. 82-86.

[16] S. Rajagopal, M. Reisslein, K. W. Ross, "Packet multiplexers with adversarial regulated traffic," IEEE Infocom'98, pp. 347-355, March 1998.

[17] J. Song and R. Boorstyn, "Efficient loss estimation in high speed networks," IEEE ATM Workshop'98, pp. 360-367, May 1998.

[18] M. Garret and W. Willinger, "Analysis, modeling and generation of self-similar VBR video traffic," ACM SIGCOMM'94, pp. 269-280, Aug. 1994.

[19] A. Lazar, G. Pacifici, and D. Pendarakis, "Modeling video sources for real time scheduling," ACM Multimedia Systems Journal, 1(6): 253-266, April 1994.

[20] H. Zhang and E. Knightly, "RED-VBR: a renegotiation-based approach to support delaysensitive VBR video," ACM Multimedia Systems Journal, 5(3): 164-176, May 1997.

[21] E. Knightly, "On the accuracy of admission control tests," IEEE ICNP'97, October 1997.

[22] B. Mark and G. Ramamurthy, "Real-time estimation of UPC parameters for arbitrary traffic sources in ATM networks," IEEE Infocom'96, pp. 384-390, March 1996.

[23] Qiang Ren and G. Ramamurthy, "A hybrid model and measurement based connection admission control and bandwidth allocation scheme for multi-class ATM networks," IEEE Globecom'98, Nov. 1998. 


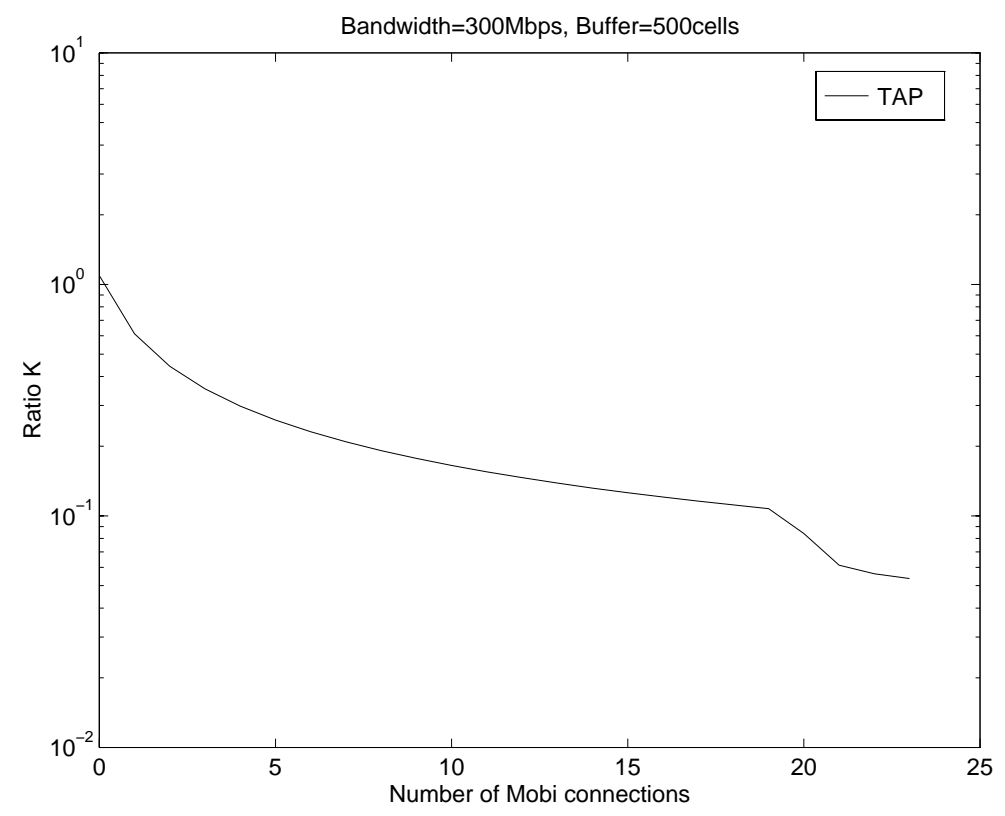

Figure 3: Effect of the number of Mobi connections on ratio K.

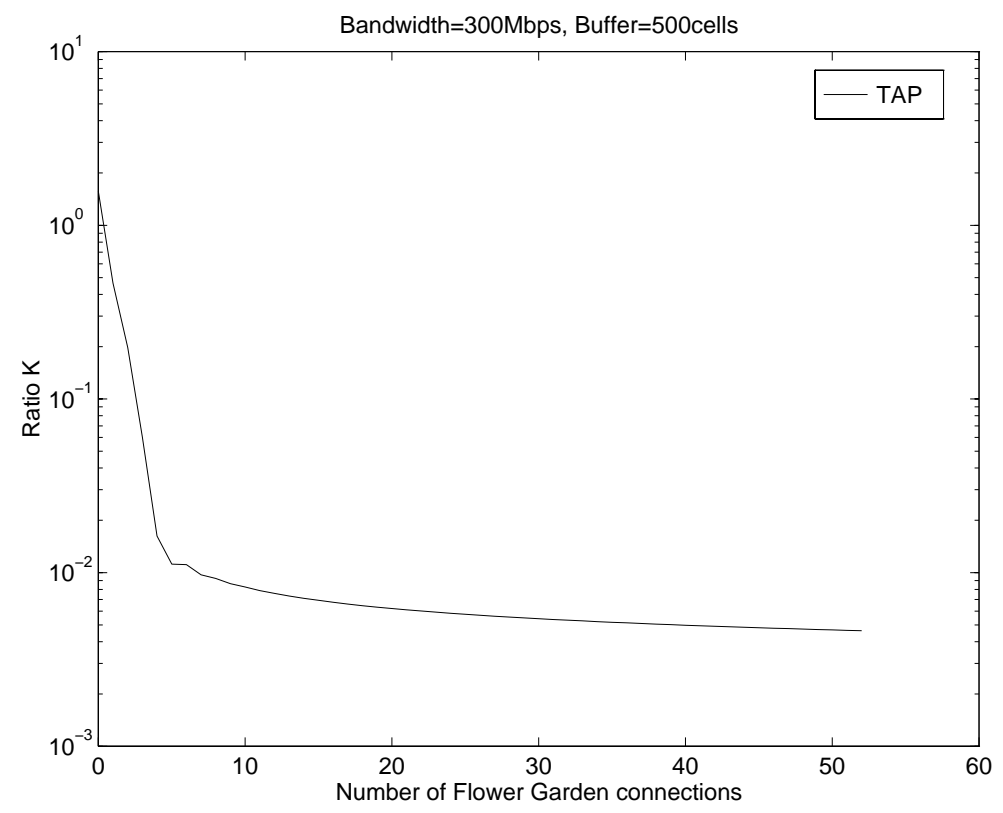

Figure 4: Effect of the number of Flower Garden connections on ratio K. 


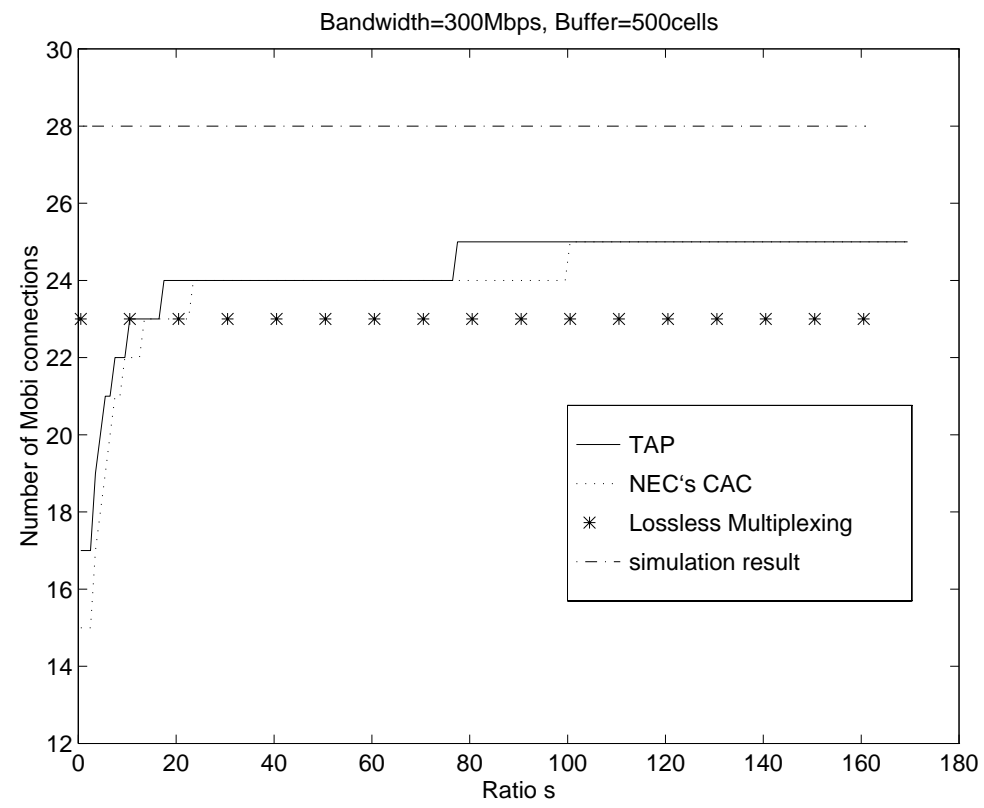

Figure 5: Effect of the ratio $s$ on the number of admissible connections for Mobi traces.

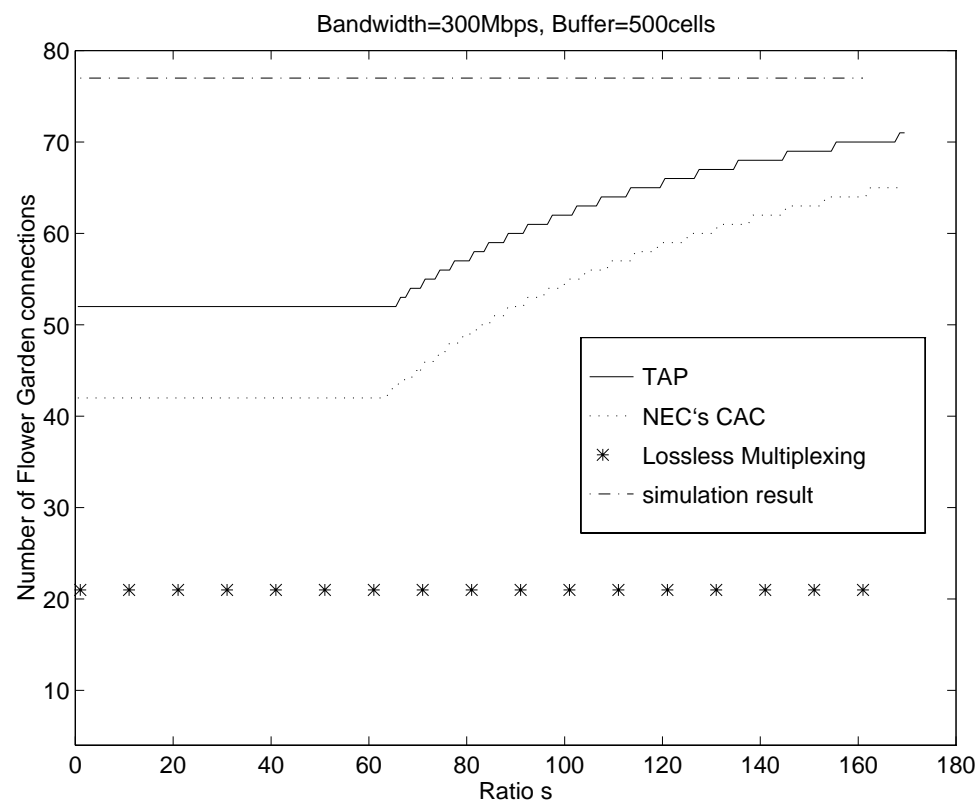

Figure 6: Effect of the ratio $s$ on the number of admissible connections for Flower Garden traces. 


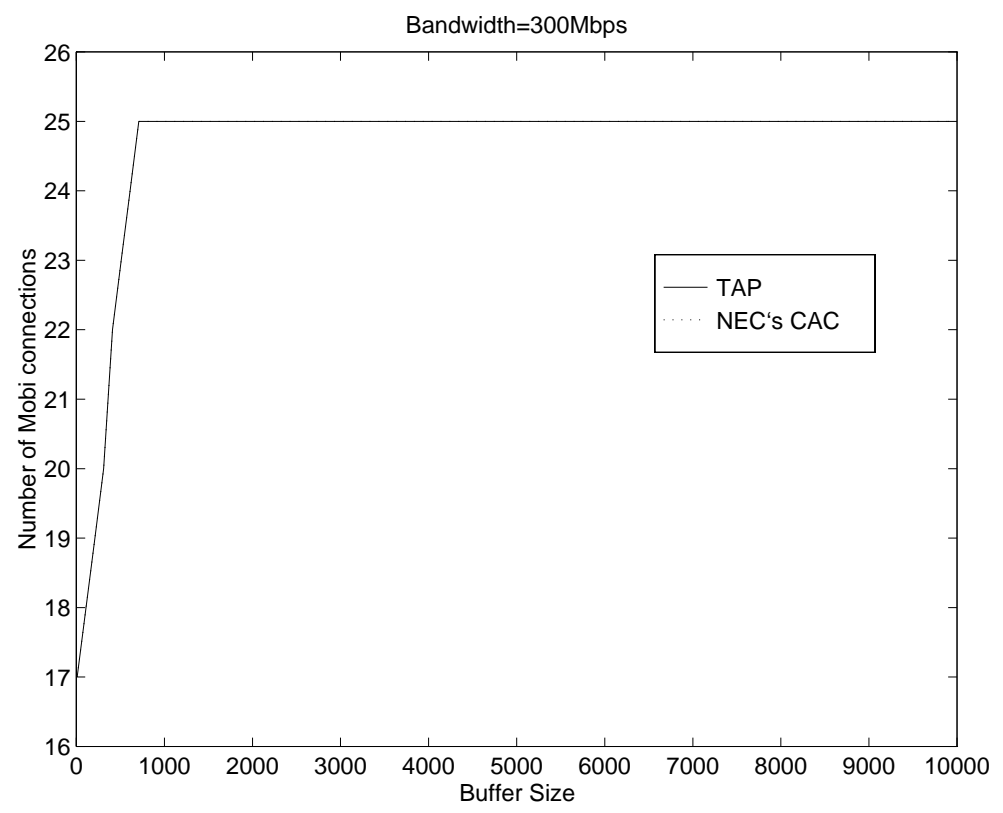

Figure 7: Effect of the buffer size on the number of admissible connections for Mobi traces.

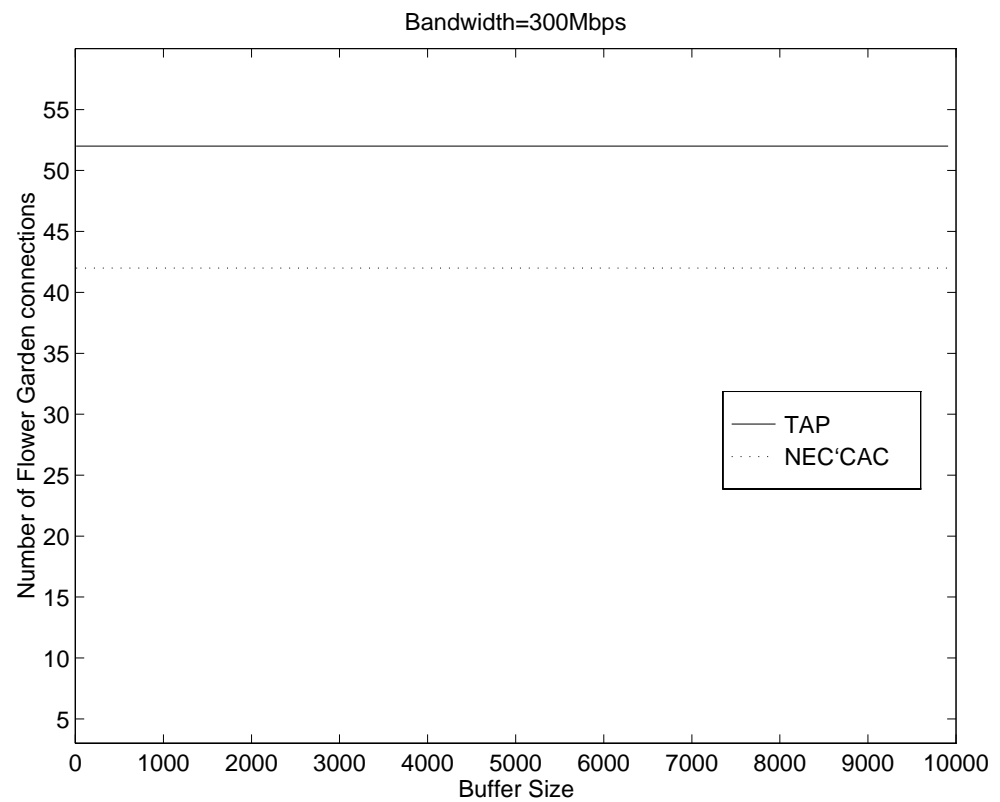

Figure 8: Effect of the buffer size on the number of admissible connections for Flower Garden traces. 


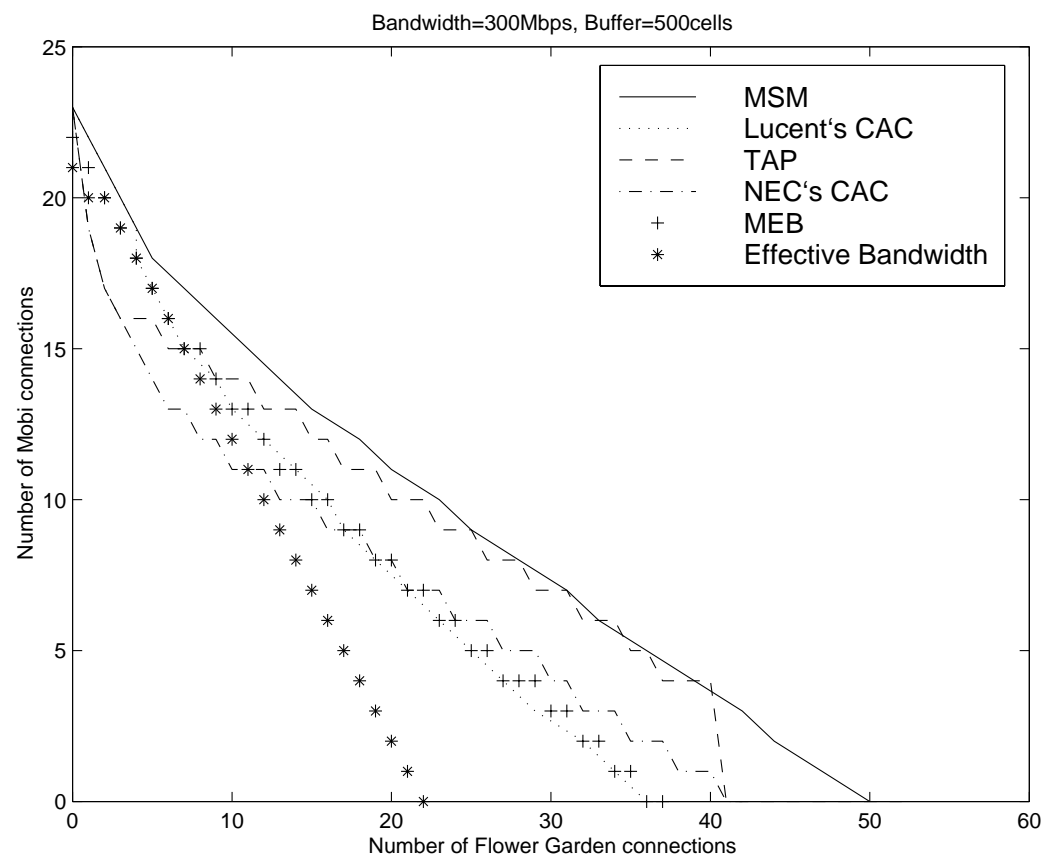

Figure 9: Admissible region for TAP, NEC's CAC, MEB, Effective Bandwidth, MSM and Lucent's CAC under $\mathrm{CLR}<10^{-5}$.

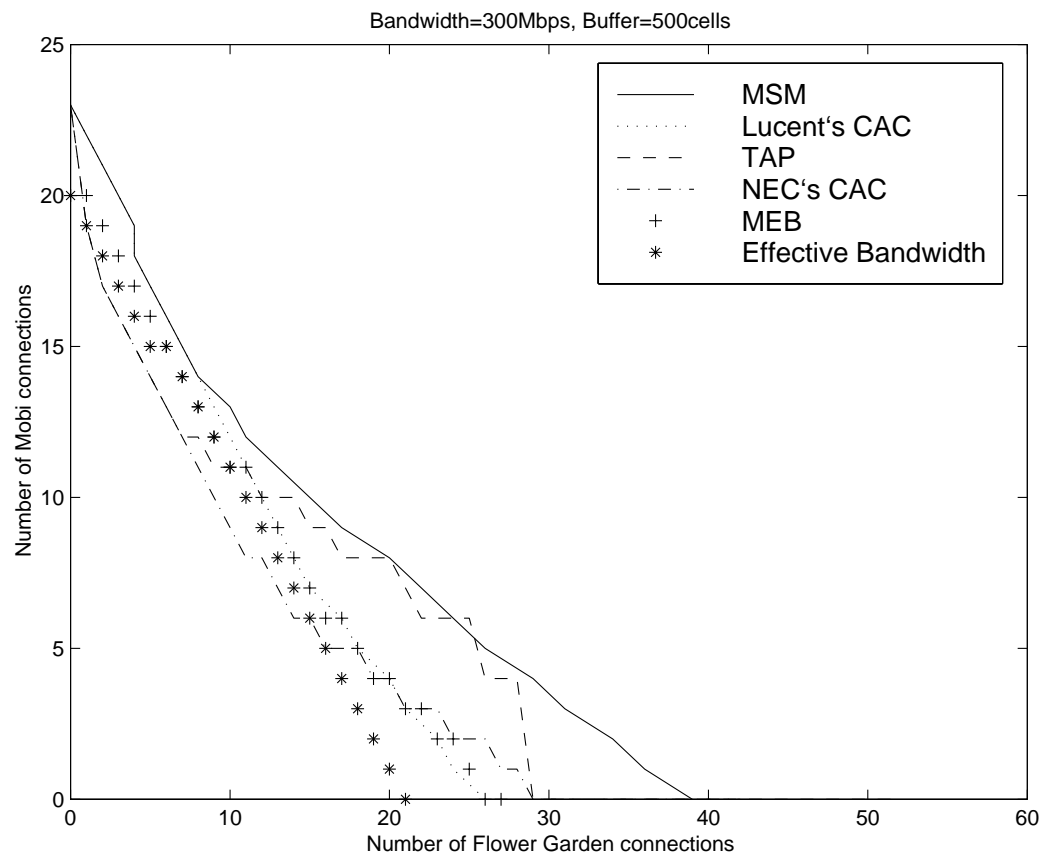

Figure 10: Admissible region for TAP, NEC's CAC, MEB, Effective Bandwidth, MSM and Lucent's CAC under CLR $<10^{-9}$. 